\title{
Phosphatase and Tensin Homolog Measurement
}

National Cancer Institute

\section{Source}

National Cancer Institute. Phosphatase and Tensin Homolog Measurement. NCI

Thesaurus. Code 147470.

The determination of the amount of phosphatase and tensin homolog protein in a biological sample. 